\title{
Utility of Simulation as a Teaching Tool for Nursing Staff Involved in Code Blue Management
}

\author{
Rithesh J D’Cunha ${ }^{1}$, Shannon F Fernandes ${ }^{2}$, Lulu Sherif $^{3} \odot$
}

\begin{abstract}
Background: The responsibility of nurses is early clinical deterioration and taking appropriate action. Knowledge and proficiency in Code Blue protocols come handy, which lacks in most nurses recognizing. Simulation-based learning plays a role in improving performance and confidence in handling such situations. This goal-based method of program evaluation aims to examine Code Blue training programs and compare them with current literature and established professional standards while assessing participants' experience and change in knowledge to this educational method.

Materials and methods: Following ethical approval, a prospective interventional study was conducted in the simulation center of a private medical college in Mangalore on 65 nursing students.

A pretest was given to the participants on the day of the session to assess their baseline understanding followed by prebriefing, all previously having received didactic lectures on Code Blue protocol, crash cart, and cardiac arrest algorithms. They were divided into three batches and each batch performed on crash cart and cardiac arrest stations using an electrocardiogram simulator. A simulated drill was enacted by the faculty on managing a Code Blue event and a discussion was followed. Nurses underwent two simulated scenarios, shockable and nonshockable cardiac arrest algorithms, followed by debriefing. Posttest and feedback form was asked to be filled.

Results: A significant increase in mean\% from pretest to posttest (55.69-77.33\%) following simulated drills.

Conclusion: The use of simulation to train nurses in Code Blue scenarios records greater satisfaction and improvement in clinical reasoning, knowledge, and skills. Hence, the incorporation of simulation teaching in training of those involved in caring for high-risk patients is the need of the hour.

Keywords: Simulation, Standardized healthcare, UG nursing students.

Indian Journal of Critical Care Medicine (2021): 10.5005/jp-journals-10071-23912
\end{abstract}

\section{INTRODUCTION}

A significant number of people succumb to sudden cardiac events the world over, despite advances in technology and knowledge as claimed by Ahern et al., ${ }^{1}$ who quotes that more than 135 million people die annually worldwide from cardiovascular events. According to Meaney et al., factors like cardiopulmonary resuscitation (CPR) quality, time of day when event occurred, and location in hospital affect survival of the hospitalized patient receiving CPR. ${ }^{2}$ While patients have the best chance at survival when nurses respond to the Code Blue call immediately, most nurses do not expect their patients to arrest. Moreover, they do not have adequate opportunities to "practice by doing" code situations. Providing high quality CPR and monitoring team performance ensure improved patient outcomes. ${ }^{2}$ Thus, standards of quality depend on well-trained staff who practice and maintain these skills. Studies have shown that attitudes of nurses influence their response in an emergency, ${ }^{3}$ and thus, implementation of training programs are necessary to improve their attitude and skills.

Simulation as a teaching strategy to improve registered nurses' performance in caring for patients is well documented in the literature. The Institute of Medicine has recommended simulation to be included as part of the training to improve patient safety. ${ }^{4}$

It is difficult to practice whatever has been learnt in classrooms directly on patients. With the help of simulators, case scenarios can be practiced in a realistic environment without the risk of medical error. Modern simulators also have a recording and feedback option making them a useful tool for student
${ }^{1,3}$ Department of Anaesthesiology, Father Muller Medical College, Mangaluru, Karnataka, India

${ }^{2}$ Department of Obstetrics and Gynaecology, Father Muller Medical College, Mangaluru, Karnataka, India

Corresponding Author: Rithesh J D'Cunha, Department of Anaesthesiology, Father Muller Medical College, Mangaluru, Karnataka, India, Phone: +91 9916253682, e-mail: ritheshcold@fathermuller.in

How to cite this article: D'Cunha RJ, Fernandes SF, Sherif L. Utility of Simulation as a Teaching Tool for Nursing Staff Involved in Code Blue Management. Indian J Crit Care Med 2021;25(8):878-880.

Source of support: Nil

Conflict of interest: None

assessment. ${ }^{5,6}$ Simulation-based learning also improves teamwork and behavioral skills necessary for trainees in a multidisciplinary healthcare system. ${ }^{7-9}$

\section{Aims and Objectives}

\section{Aims}

The aim of this study was to evaluate the efficacy of a simulationbased training program for nursing staff involved in Code Blue.

\section{Objectives}

- To train the nursing students to recognize and manage cardiac arrest cases using simulation 
- To evaluate the acceptance of simulation by the participants using a satisfaction questionnaire.

- To record the change in knowledge and attitude following training using pretest and posttest multiple-choice questions.

\section{Materials and Methods}

A prospective interventional study was conducted for 65 finalyear nursing students over a period of 3 months from October 2019 to December 2019. Three sessions of 3-hour duration were conducted, and each session had a batch of 20-25 nurses. Each session was facilitated by the same set of three American Heart Association (AHA)-certified instructors who were not a part of the investigator team. Single group pretest/posttest method was used with convenience sampling.

Following ethical clearance, informed consent, and validation of the questionnaires and test questions by the members of the Code Blue Committee, the sessions were rehearsed through dry runs by the nursing faculty.

On the day of the session, each participant nurse answered the pretest that assessed their knowledge and attitude on Code Blue protocol, crash cart, and cardiac arrest algorithm based on AHA guidelines. This was followed by a didactic lecture on Code Blue protocol, crash cart, and cardiac arrest algorithms. The nurses then participated in small group hands on stations on the crash cart and cardiac arrest algorithms using an electrocardiogram simulator. The nursing participants then observed a Code Blue simulation scenario of 15-minute duration enacted by the facilitators. The debrief session following this aimed at clarifying the concepts and queries to the participants. The nurses were then divided into two groups, the first group participated in the first simulated scenario on shockable rhythm algorithm, while the other group observed. This was followed by debriefing for both the groups. For the second scenario on nonshockable rhythm algorithm, the second group participated, while the first group observed. This was again followed by a debriefing session. The plus-delta method was used for debriefing. Both the scenarios followed by debriefing were completed within 90 minutes. The session was concluded once the nurses answered the posttest, which was a shuffled order of the pretest questions to assess the learning. They also answered a feedback questionnaire that was aimed at capturing their attitude toward this teaching and learning modality.

\section{Statistical Analysis}

Cronbach's alpha was used to analyze the reliability of the questionnaire used to record participant satisfaction, and the average of pretest and posttest was compared using the Student's t-test.

\section{Results}

The following study was conducted in the Father Muller Simulation Center among 65 nursing students.

Of the total, $12.3 \%$ of the students were males and $87.7 \%$ were females.

Only eight students (12.3\%) had previously heard of simulation as a novel teaching method (Table 1).

The experience of participants toward simulation was sought, and opinions were taken for improvement of the teaching program:

$80 \%$ of the students identified simulation as an innovative form of learning;

95.4\% concluded that simulation significantly contributed to skill development and is required as part of training before handling patients directly;

92.3\% found debriefing useful in analyzing errors done and noted that it helped to reinforce the didactic method of teaching; and $23.07 \%$ of the students felt stressed during the scenario (Table 2).

Some suggestions that came forth were as follows:

- It would be better if the scenario was run in the wards rather than in the simulation center.

- Active participation of all students must be ensured.

- Helpful to run other code scenarios also.

- Need for more training in analyzing cardiac arrest rhythms.

Table 1: Comparison of pretest and posttest results

\begin{tabular}{|c|c|c|c|c|c|c|c|}
\hline & $N$ & Mean & Std. deviation & Mean (\%) & Mean difference (\%) & t test & $p$ value \\
\hline Pretest & 65 & 8.35 & 2.58 & 55.69 & 38.86 & 0.000 & Highly significant \\
\hline Posttest & 65 & 11.60 & 2.01 & 77.33 & & & \\
\hline
\end{tabular}

Table 2: Responses based on feedback form

\begin{tabular}{|c|c|c|c|c|c|}
\hline Parameter & Strongly agree (No, \%) & Agree & Neutral & Disagree & Strongly disagree \\
\hline $\begin{array}{l}\text { This experience will improve my performance in } \\
\text { the actual clinical setting }\end{array}$ & $60(92.3 \%)$ & $5(7.7 \%)$ & - & - & - \\
\hline This simulation was a valuable learning experience & $52(80 \%)$ & $11(16.93 \%)$ & $2(3.07 \%)$ & - & - \\
\hline Debriefing was a valuable learning experience. & $60(92.3 \%)$ & $5(7.7 \%)$ & - & - & - \\
\hline The program goals and objectives were clearly met. & $58(89.23 \%)$ & $5(7.7 \%)$ & $2(3.07 \%)$ & - & - \\
\hline $\begin{array}{l}\text { This training is vital and needs to be included in the } \\
\text { nursing curriculum. }\end{array}$ & $62(95.38 \%)$ & - & $3(4.62 \%)$ & - & - \\
\hline It helped fill gaps in present skill/attitude. & $62(95.38 \%)$ & $3(4.62 \%)$ & - & - & - \\
\hline Satisfied with teaching tools and faculty. & $60(92.3 \%)$ & $5(7.7 \%)$ & & & \\
\hline
\end{tabular}




\section{Discussion}

The effectiveness of using simulation to enhance the knowledge of nurses, their confidence levels, and performance when a patient's clinical condition begins to deteriorate in the ward has been evaluated in several studies from academic and practice settings. In order to examine the ability of nursing students to assess, identify, and respond to patients whose clinical condition was deteriorating in a simulated environment, Cooper et al. ${ }^{10}$ used a mixed methods design.

With the help of pretest and posttest, Lindsey and Jenkins ${ }^{11}$ performed a randomized experimental study to identify the impact of a clinical simulation educational intervention on the clinical judgment of student nurses in managing patients experiencing rapid clinical deterioration. Those who received the simulation training had significantly higher posttest scores $(M=90.91$, $\mathrm{SD}=8.73)$ than those who had not received the intervention $(M=64.80, S D=19.69), t(77)=7.65, p<0.001)$. Their results support the use of simulation as an educational intervention to enhance knowledge and performance levels in rapid response systems. Our study showed a significantly higher posttest score. We, however, did not use a control group to compare.

Liaw et al. ${ }^{12}$ did a study on the opinion of nursing students toward simulation-based learning in helping them prepare to transfer knowledge to clinical practice when they encountered deteriorating patients.

Utilizing a pre- and postintervention study, Askew et al. ${ }^{13}$ support simulation as an educational tool to improve knowledge, skills, confidence, and communication while working with patients whose clinical condition is deteriorating. Their study was performed to improve the ability of a registered nurse to recognize and respond to rescue situations in a surgical oncology unit.

\section{Conclusion}

Clinical experience is complemented by simulation when used as an educational tool and serves as an effective teaching and learning tool for adult learners. The use of simulation to train nurses in Code Blue scenarios reports greater satisfaction, improvement in clinical reasoning, and enhances knowledge and skills. Hence, simulation training needs to be incorporated in the regular training programs to improve patient safety. Our study had a few limitations. Our sample size was small as convenience sampling was used and only nurses from our hospital were included, making it difficult to generalize the findings. A larger study including nurses from other setups would be ideal to confirm our findings. In our study, the participant's reaction and change in knowledge to this educational intervention were recorded. The change in behavior (Kirkpatrick level 3 ) and result on patient care (Kirkpatrick 4) were not recorded. A longer period of follow-up is required to record these findings and know the effectiveness of this program with the ultimate goal of improving patient safety.

\section{OrCID}

Rithesh J D'Cunha 으 https://orcid.org/0000-0002-8483-4787

Shannon F Fernandes $\odot$ https://orcid.org/0000-0001-9054-0104

Lulu Sherif 10 https://orcid.org/0000-0001-6437-4464

\section{References}

1. Ahern RM, Lozano R, Naghavi M, Foreman K, Gakidou E, Murray CJ. Improving the public health utility of global cardiovascular mortality data: the rise of ischemic heart disease. Popul Health Metr 2011;9(1):8. DOI: 10.1186/1478-7954-9-8.

2. Meaney PA, Bobrow BJ, Mancini ME, Christenson J, de Caen AR, Bhanji F, et al. CPR quality: Improving cardiac resuscitation outcomes both inside and outside the hospital: a consensus statement from the American Heart Association. Circulation 2013;128(4):1-19. DOI: 10.1161/CIR.0b013e31829d8654.

3. Dwyer T, Mosel WL. Nurses' behavior regarding CPR and the theories of reasoned action and planned behavior. Resuscitation 2002;52(1):85-90. DOI: 10.1016/s0300-9572(01)00445-2.

4. Kohn LT, Corrigan JM, Donaldson MS, editors. Institute of Medicine (US) Committee on Quality of Health Care in America. To err is human: building a safer health system. Washington (DC): National Academies Press (US); 2000. PMID: 25077248.

5. Okuda $Y$, Quinones J. The use of simulation in the education of emergency care providers for cardiac emergencies. Int J Emerg Med 2008;1(2):73-77. DOI: 10.1007/s12245-008-0034-2.

6. Gaba DM. The future vision of simulation in health care. Qual Saf Health Care 2004;13(Suppl. 1):i2-i10. DOI: 10.1136/qhc.13.suppl_1.i2.

7. Weller JM, Nestel D, Marshall SD, Brooks PM, Conn JJ. Simulation in clinical teaching and learning. Med J Aust 2012;196(9):594. DOI: 10.5694/mja10.11474.

8. Cook DA, Hatala R, Brydges R, Zendejas B, Szostek JH, Wang AT, et al. Technology-enhanced simulation for health professions education: a systematic review and meta-analysis. JAMA 2011;306(9):978-988. DOI: 10.1001/jama.2011.1234.

9. Cook DA, Hamstra SJ, Brydges R, Zendejas B, Szostek JH, Wang AT, et al. Comparative effectiveness of instructional design features in simulation-based education: systematic review and meta-analysis. Med Teach 2013;35(1):e867-e898. DOI: 10.3109/0142159X.2012.714886.

10. Cooper S, Kinsman L, Buykx P, McConnell-Henry T, Endacott R, Scholes J. Managing the deteriorating patient in a simulated environment: nursing students' knowledge, skill and situation awareness. J Clin Nurs 2010;19(15/16):2309-2318. DOI: 10.1111/j.13652702.2009.03164.x.

11. Lindsey $P L$, Jenkins S. Nursing students' clinical judgment regarding rapid response: the influence of a clinical simulation education intervention. Nurs Forum 2013;48(1):61-70. DOI: 10.1111/nuf.12002.

12. Liaw S, Chan S, Scherpbier A, Rethans J, Pua G. Recognizing, responding to and reporting patient deterioration: transferring simulation learning to patient care settings. Resuscitation 2012;83(3):395-398. DOI: 10.1016/j.resuscitation.2011.08.021.

13. Askew T, Trotter TL, Vacchiano S, Garvey P, Overcash J. Avoiding failure to rescue situations: a simulation exercise for oncology nurses. Clin J Oncol Nurs 2012;16(5):530-532. DOI: 10.1188/12.CJON.530-532. 